\title{
Acquisition Duration
}

National Cancer Institute

\section{Source}

National Cancer Institute. Acquisition Duration. NCI Thesaurus. Code C69269.

The amount of time elapsed during the capture of data. 